\title{
Influence of periodic pressure on dendritic morphology and sidebranching
}

\author{
Shan Shang ${ }^{1,2}$, Zhi-peng Guo ${ }^{3}$, Zhi-qiang Han ${ }^{3}$, ${ }^{*}$ Xin-yu Zhang ${ }^{1,2}$, *Yi-nuo Cheng ${ }^{4}$, Jun Li ${ }^{1,2}$ \\ 1. State Key Laboratory of Automotive Safety and Energy, Tsinghua University, Beijing 100084, China \\ 2. School of Vehicle and Mobility, Tsinghua University, Beijing 100084, China \\ 3. School of Materials Science and Engineering, Tsinghua University, Beijing 100084, China \\ 4. HiRain Technologies Co., Ltd., Beijing 100191, China
}

\begin{abstract}
The influence of periodic pressure with low and high frequencies on microstructure and dendritic sidebranching was studied by using 3-D phase field method. In both low and high frequency cases, the variation trend of SDAS (secondary dendritic arm spacing) with increasing pressure frequency is opposite to that of sidebranching frequency, while the variation trend of the average length of secondary arms is consistent with that of sidebranching frequency. The high sidebranching frequency indicates that more secondary arms share the whole driving force of dendrite growth, resulting in lower driving force for each one and leading to less developed secondary arms. The smallest SDAS is obtained when perturbed by the periodic pressure with the frequency of $0.157 / \tau_{0}\left(\tau_{0}\right.$ is the physical unit of time in the dimensionless phase field model) and $2.200 / \tau_{0}$ in low and high frequency cases, respectively. Comparisons of dendritic morphology and secondary arms are made between the low and high frequency cases. Firstly, in the low frequency case, secondary arms are luxuriant especially when pressure frequency is low, with many high-order side branches stretching out. Secondly, the average length of secondary arms in primary dendrite is longer in the low frequency case than that without pressure, and much longer than that in the high frequency case. Thirdly, the dendrite tip without side branches in the high frequency case is much longer than that in the low frequency case. All of the differences in dendritic morphology and sidebranching in the two cases can be attributed to the different modulation mechanism. In the low frequency case, periodic pressure determines tip velocity and then modulates sidebranching directly. While in the high frequency case, periodic pressure cannot determine sidebranching directly, but via modulating tiny protuberances in dendrite tip, part of which evolves into side branch. In this case, the tiny protuberances take part of the whole driving force, leading to less developed secondary arms.
\end{abstract}

Key words: periodic pressure; dendritic morphology; SDAS; phase field method

CLC numbers: TP391.9 Document code: A

Article ID: 1672-6421(2020)04-279-07

$\mathrm{D}$ endritic growth behavior with side branches behind a dendrite tip is of great importance in the solidification process, which has significant influence on final microstructure and mechanical properties. It has been proved that pressure is an effective external perturbation to modulate dendritic growth and change sidebranching behavior ${ }^{[1-10]}$. Lacombe et al. ${ }^{[4-6]}$ studied the effect of pressure on succinonitrile dendrite. According to the Clapeyron effect, the equilibrium melting temperature altered with the change of pressure, resulting in that the

\section{*Xin-yu Zhang}

Male, Master, Senior Engineer. His research interests mainly focus on the intelligent vehicles and robotics, machine learning, deep learning, and driving safety.

E-mail: xyzhang@tsinghua.edu.cn

**Yi-nuo Cheng

E-mail: yinuo.cheng@hirain.com

Received: 2020-02-26; Accepted: 2020-05-29 velocity and radius of dendrite tip transformed from initial steady states to states appropriate for the new thermal conditions. Moreover, a dominant side branch was formed, due to the destabilized dendrite interface caused by pressure change.

The effect of periodic pressure on dendritic growth has been studied experimentally and numerically by using the phase field method ${ }^{[11-15]}$. Phase field method ${ }^{[16-23]}$ is a powerful tool to study dendritic growth and sidebranching during pressurized solidification ${ }^{[14,15,24-26]}$. Börzsönyi et al. ${ }^{[14,15]}$ studied the effect of pressure on melting point in phase field model, and found that oscillating pressure or heating can tune the frequency of dendritic sidebranching dramatically. The underlying mechanism for dendritic sidebranching has been explored by applying periodic pressures at different frequencies using 3-D phase field modeling ${ }^{[25]}$. It is revealed that if the frequency of pressure is lower than the critical 
value, both the tip velocity and the sidebranching would be completely synchronized with the perturbation. Whereas, if the perturbation frequency is higher than the critical value, rather than increasing, the sidebranching frequency would become stable and maintain at the same magnitude as that of the natural sidebranching, i.e., when no external perturbation was applied.

Nevertheless, the influence of periodic pressure on final microstructure and dendritic morphology, such as the size of secondary arms and SDAS which are associated with subsequent mechanical properties, has not been studied yet.

In this work, the dendritic morphology and sidebranching behavior under periodic pressure with low and high frequencies were studied using the 3-D phase field method. The variation trends of sidebranching frequency, SDAS and the size of secondary arms with increasing pressure frequency were calculated and analyzed. The distinctions of dendritic morphology and secondary arms were found between the low and high frequency cases, and the reason associated with modulation mechanism was revealed.

\section{Model description}

The 3-D phase field model has been described in detail in Ref. [25]. The 3-D controlling equations (anisotropic) for phase field and solute field ${ }^{[18,19,27]}$ are as follows:

$$
\begin{aligned}
& \tau \frac{\partial \Phi}{\partial t}=\nabla \cdot\left(W(\vec{n})^{2} \nabla \Phi\right)+\frac{\partial}{\partial x}\left(|\nabla \Phi|^{2} W(\vec{n}) \frac{\partial W(\vec{n})}{\partial \Phi x}\right) \\
& +\frac{\partial}{\partial y}\left(|\nabla \Phi|^{2} W(\vec{n}) \frac{\partial W(\vec{n})}{\partial \Phi y}\right)+\frac{\partial}{\partial z}\left(|\nabla \Phi|^{2} W(\vec{n}) \frac{\partial W(\vec{n})}{\partial \Phi z}\right) \\
& +\Phi\left(1-\Phi^{2}\right)-\lambda\left(1-\Phi^{2}\right)^{2}(\theta+k U)
\end{aligned}
$$

$$
\left(\frac{1+k}{2}-\frac{1-k}{2}\right) \frac{\partial U}{\partial t}=\nabla \cdot\left(D \frac{1-\Phi}{2} \nabla U-\vec{J} a t\right)+\frac{1}{2}[1+(1-k) U] \frac{\partial \Phi}{\partial t}
$$

where $\tau$ is the relaxation time, $\Phi$ is phase field which is -1 in bulk liquid and 1 in bulk solid, $W(\vec{n})$ is the anisotropic width of the diffuse interface with $\vec{n}$ the unit normal vector pointing out into the liquid, $k$ is the partition coefficient for solute, and $D$ is solute diffusivity. $(\theta+k U)$ is the driving source term, which is related to the undercooling of the system. $\lambda$ is the scaling parameter and defined as:

$$
\lambda=\frac{15}{16} \frac{R T_{\mathrm{M}}(1-k)}{v_{0} H|m|} \Delta T_{0}
$$

where $R$ is the gas constant, $T_{\mathrm{M}}$ is the melting temperature of the solvent, $v_{0}$ is the molar volume, $H$ is the energy barrier of the double well potential, and $m$ is the liquidus slope in the phase diagram.

$$
\Delta T_{0}=\frac{|m| c_{\infty}(1-k)}{k}
$$

is the equilibrium freezing temperature range according to $c_{\infty}$, where $c_{\infty}$ is the initial solute concentration.

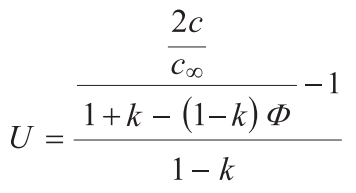

and

$$
\theta=\frac{T-T_{\mathrm{M}}-m c_{\infty}}{\Delta T_{0}}
$$

are dimensionless solute concentration and temperature, respectively, where $c$ is molar solute concentration.

To consider the influence of periodic pressure, a pressure undercooling, $\xi$, caused by periodic pressure according to the classical Clausius-Clapeyron equation was added into the source term $(\theta+k U)$ of the governing equations for phase field. $\xi$ is in the form of sinusoidal wave shown as follows:

$$
\xi=\xi_{0} \sin \left(2 \pi f_{\xi} t\right)
$$

where $f_{\xi}$ represents the frequency of pressure, $\xi_{0}$ is the amplitude of pressure undercooling. The source term acted as the driving force for the dendrite growth is $F_{\text {driving }}=-(\theta+k U+\xi)$, which is associated with the whole undercooling caused by temperature, solute and pressure.

In this work, a solid seed was placed at the bottom corner of the simulation domain. $\theta$ was set to be homogeneous $(\theta=-0.12)$ across the domain, about $5 \mathrm{~K}$ of undercooling for the Al-3wt.\% $\mathrm{Cu}$ alloy. The amplitude of periodic pressure $\xi_{0}$ was set to be 0.05 , about $2.08 \mathrm{~K}$ of pressure undercooling for this alloy. A total of 60,000 time steps were calculated for each simulation. Other parameters in these equations, simulation parameters, and the crystal anisotropy were set the same as in Ref. [25]. Adaptive mesh refinement and parallel computing were adopted in this work to solve the governing equations ${ }^{[27]}$.

\section{Simulation results and discussion}

\subsection{Dendritic morphology without pressure}

Solidification microstructure and dendritic morphology of Al-3wt.\% Cu alloy without employing any pressure were investigated. Figure 1 shows the secondary dendritic arms (i.e., side branches) of $1 / 8$ grain at 60,000 time steps. The secondary dendritic arms grow from a primary dendrite, with smaller arms nearby the dendrite tip and larger ones away from the dendrite tip since they grow for a longer time. This phenomenon is called dendritic sidebranching, and secondary arms are also called side branches.

The frequency of sidebranching $f_{\mathrm{s}}$ without pressure (hereinafter referred to as unperturbed sidebranching) is 


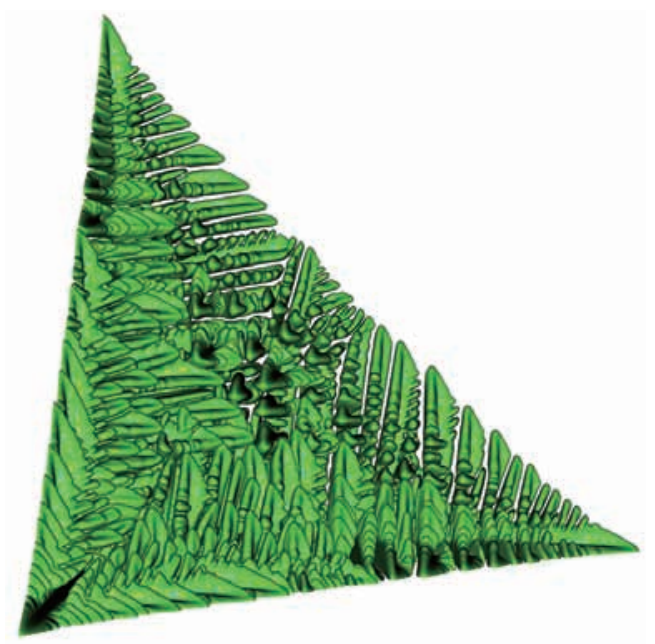

Fig. 1: Dendrite morphology without pressure at 60,000 time steps

calculated by using the equation as follows ${ }^{[28,29]}$ :

$$
f_{\mathrm{s}}=\bar{v} / \bar{\lambda}_{2}
$$

where $\bar{v}$ is the average value of tip velocity and $\bar{\lambda}_{2}$ is the average wavelength (average secondary dendrite arm spacing, i.e., SDAS). The value of $\bar{\lambda}_{2}$ or SDAS is calculated as:

$$
\bar{\lambda}_{2}=\mathrm{SDAS}=\lambda_{n} /(n-1)
$$

where $\lambda_{n}$ is the length of the line drawn from center to center of the measured $n$ side branches, and $n$ is the number of side branches close to the tip, as shown in Fig. 2.

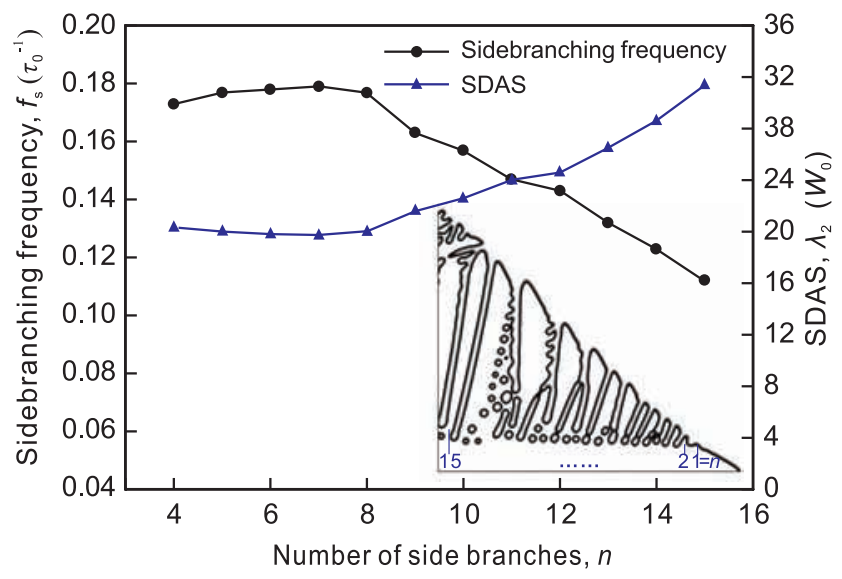

Fig. 2: Frequency of sidebranching and SDAS as a function of the number of measured side branches $n$

In the dimensionless phase field model, the physical units of length and time are $W_{0}$ and $\tau_{0}$, respectively. Some dimensionless variables in this study, such as frequency, the length of side branches and SDAS, are related to these two physical units. The definitions of $W_{0}$ and $\tau_{0}$ are as follows:

and

$$
\begin{gathered}
W_{0}=\lambda d_{0} / a_{1} \\
\tau_{0}=d_{0}{ }^{2} a_{2} \lambda^{3} /\left(D a_{1}^{2}\right)
\end{gathered}
$$

$$
d_{0}=\Gamma / \Delta T_{0}
$$

is the chemical capillary length, where $\Gamma$ is the Gibbs-Thomson coefficient which is determined by materials, $a_{1}=0.8839$ and $a_{2}=0.6267$ according to the thin interface limit analysis ${ }^{[27]}$.

The frequency of sidebranching and SDAS without pressure are calculated with different $n$ based on the above methods. As shown in Fig. 2, for a certain primary dendrite, the sidebranching frequency decreases monotonically with the increase of the number of measured sidebranches $n$. Side branches formed early and farther away from the dendrite tip are larger due to the coarsening process, so the sidebranching frequency decreases with the increase of $n$. However, SDAS is negatively correlated with the side branching frequency, so the SDAS varies inversely with the number of measured side branches $n$. The frequency range of unperturbed sidebranching is around $0.112 / \tau_{0}-0.179 / \tau_{0}$ (scaled). Since the final SDAS which is calculated considering almost all the sidebranches in a certain primary dendrite is associated with mechanical properties, the final SDAS and sidebranching frequency under pressure were studied.

\subsection{Dendritic morphology under periodic pressure}

The dendritic growth and sidebranching under periodic pressure with different frequencies, including low frequency and high frequency, were studied. In the low frequency case, the magnitude of pressure frequency is almost the same as that of unperturbed sidebranching frequency $\left(0.112 / \tau_{0}-0.179 / \tau_{0}\right)$. In the high frequency case, the magnitude of pressure frequency is much higher than that of unperturbed sidebranching frequency. The pressure frequency in the high frequency case is about ten times higher than that in the low frequency case.

The dendritic morphology and sidebranching with four different periodic pressure at low frequency is shown in Fig. 3. Firstly, when the pressure frequency is relatively low $\left(0.044 / \tau_{0}\right)$, side branches are sparse and large, and the higherorder side branches are well developed. Secondly, when the pressure frequency increases and is in the range of unperturbed sidebranching frequency, such as $0.122 / \tau_{0}$ and $0.157 / \tau_{0}$, more side branches stretch out regularly and densely. Sidebranching frequency increases with the frequency of pressure, as illustrated in Fig. 4. However, when pressure frequency is relatively high, such as $0.220 / \tau_{0}$, sidebranching frequency does not increase with pressure any more.

The final SDAS with low frequency of pressure is shown in Fig. 4. When the frequency of pressure increases, the SDAS declines firstly, and then rises. The smallest SDAS is obtained when the frequency of pressure is $0.157 / \tau_{0}$. Apparently, the variation trend of sidebranching frequency is contrary with that of SDAS.

In addition to SDAS, attention should also be paid to the size of secondary arms. Based on the dendrite arms shown in Fig. 3, the average length of all side branches in the primary dendrite is calculated and illustrated in Fig. 5. It can be seen that the variation trend of the average length of a side branch is consistent with that of SDAS and inverse to that of sidebranching frequency. When pressure frequency is $0.157 / \tau_{0}$, 

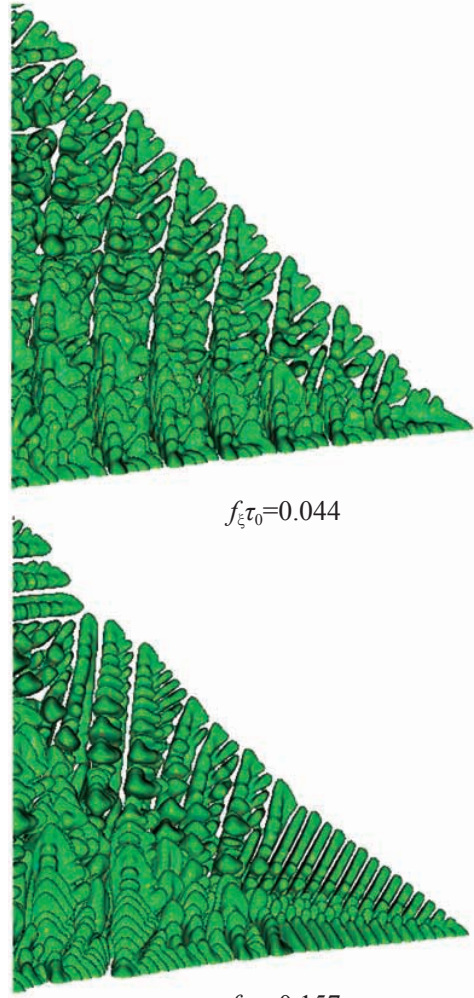

$f_{\xi} \tau_{0}=0.157$

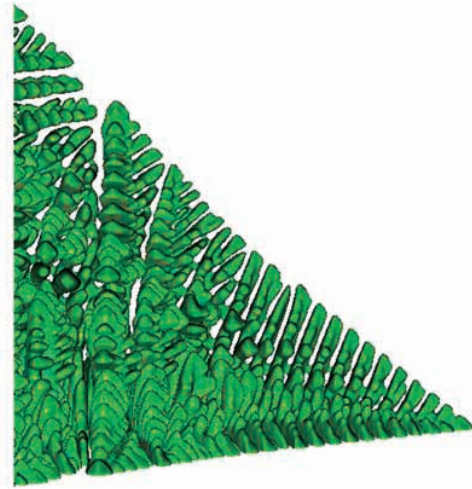

$f_{\xi} \tau_{0}=0.122$

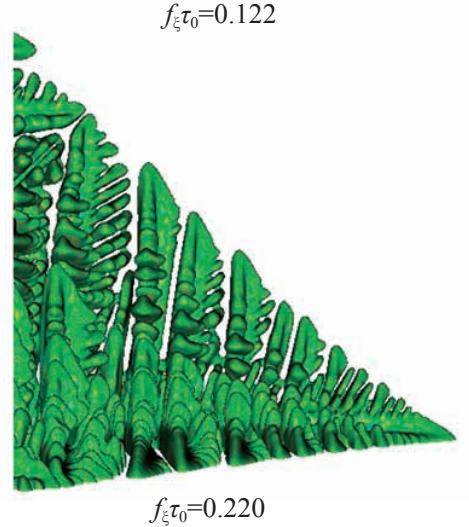

Fig. 3: Dendritic morphology and sidebranching with low frequency periodic pressure

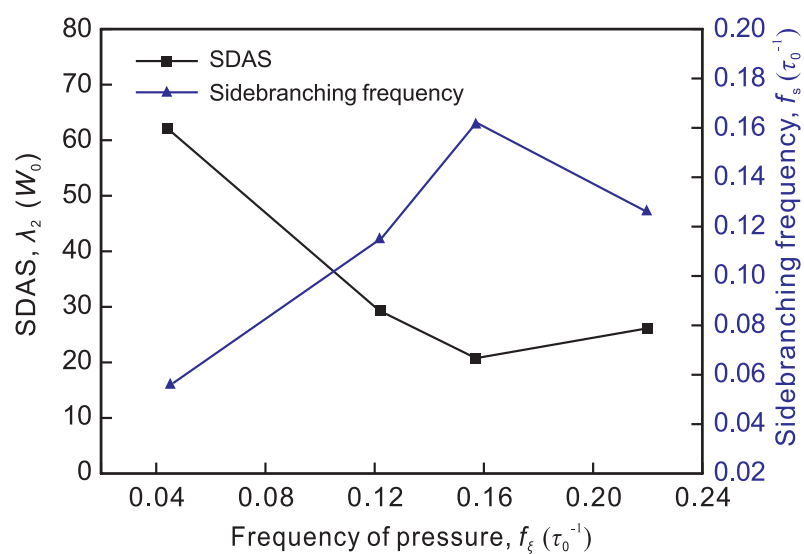

Fig. 4: Variation trend of SDAS with low frequency periodic pressure

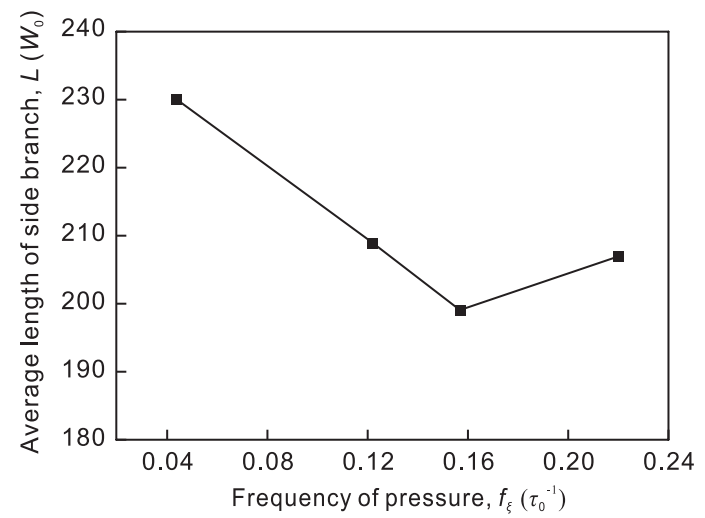

Fig. 5: Average length of side branch with low frequency periodic pressure the sidebranching frequency is highest while the average length of side branch is shortest in the low frequency case. Since more secondary arms grow in the primary dendrite, which share the whole driving force of dendrite growth $\left(F_{\text {driving }}=-(\theta+k U+\xi)\right)$, there is lower driving force for the growth of each secondary arm. This is the reason why side branches are shorter when sidebranching frequency is higher. In a word, the average length of secondary arms increases with the decrease of the number of secondary arms in primary dendrite, the variation trend of the average length of secondary arms is opposite to that of sidebranching frequency, as well as the frequency of pressure.

The effect of periodic pressure with high frequency (about 2.0/ $\tau_{0}$ magnitude) on dendrite morphology was also studied. As shown in Fig. 6, the dendritic morphology and sidebranching phenomenon are different with the increase of the pressure frequency. Secondary arms become denser with increasing pressure frequency when $f_{\xi}<4.400 / \tau_{0}$. However, when $f_{\xi}=4.400 / \tau_{0}$, sidebranching does not follow this variation trend as if it is not influenced by the periodic pressure.

The final SDAS under periodic pressure with high frequencies is shown in Fig. 7. When the frequency of pressure increases from $1.100 / \tau_{0}$ to $4.400 / \tau_{0}$, the SDAS declines firstly, and then rises, and the smallest SDAS $\left(23.6 W_{0}\right)$ is obtained when the frequency of pressure is $2.200 / \tau_{0}$. The same as in the case of low frequency, the variation trend of SDAS with the frequency of pressure is on the contrary with that of sidebranching frequency, while the variation trend of average length of secondary arms is consistent with that of sidebranching frequency, as shown in Fig. 8. 


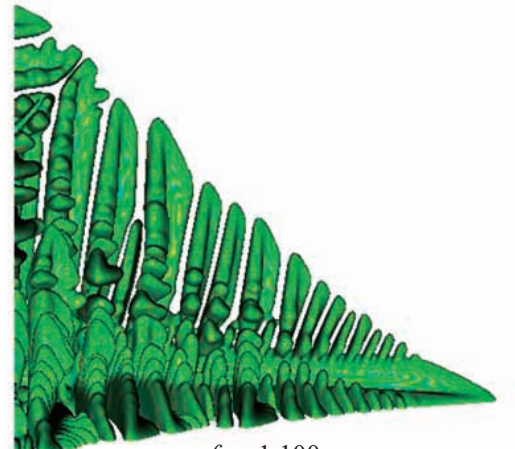

$f_{\varepsilon} \tau_{0}=1.100$

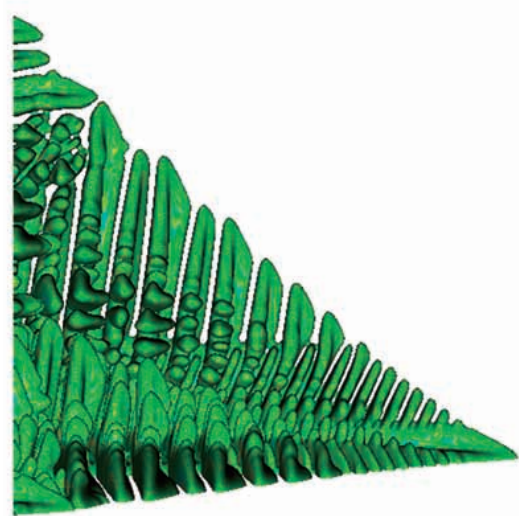

$f_{\tilde{\xi}} \tau_{0}=2.200$
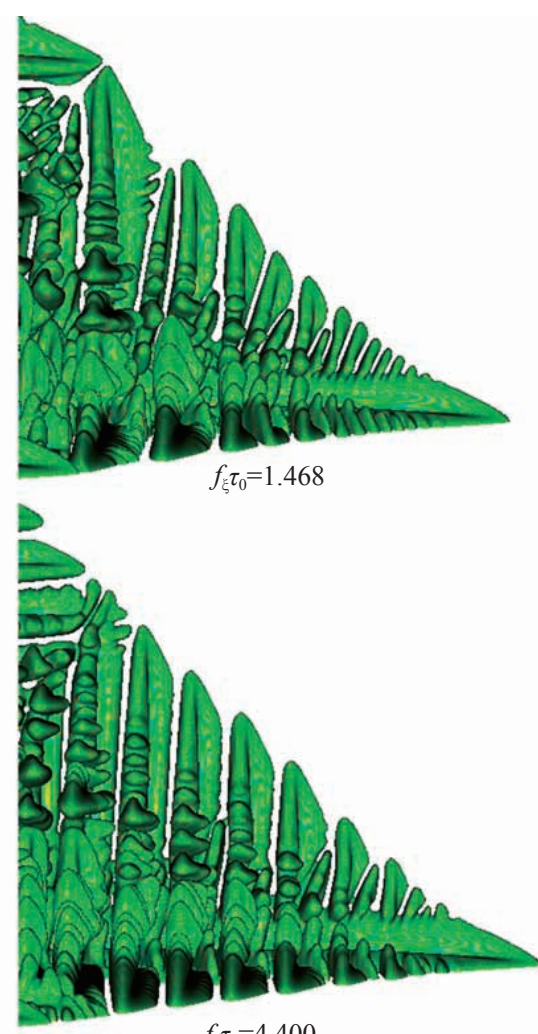

$f_{\varepsilon} \tau_{0}=4.400$

Fig. 6: Dendritic morphology and sidebranching with high frequency periodic pressure

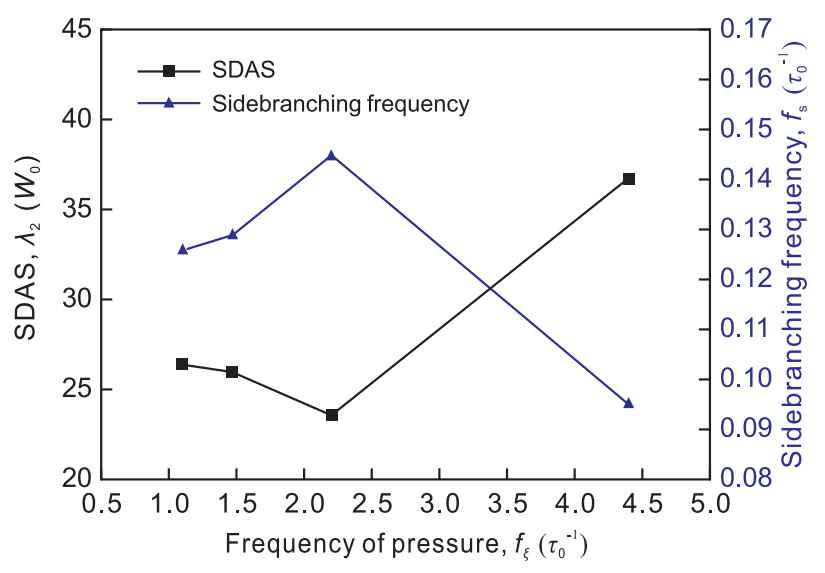

Fig. 7: Variation trend of SDAS with high frequency periodic pressure

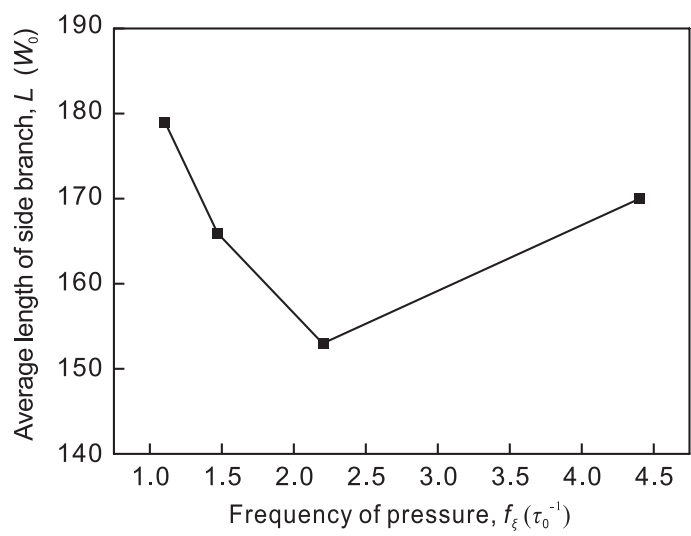

Fig. 8: Average length of side branch with high frequency periodic pressure

\subsection{Comparison between the two cases}

Based on the above analysis, the dendritic morphologies are quite different under low frequency and high frequency periodic pressure modulation. The difference in morphology can be seen by comparing Fig. 3 and Fig. 6, as well as Fig. 9. Firstly, in low frequency, secondary arms are luxuriant especially when pressure frequency is low, with more high-order side branches stretching out than that without perturbation, while secondary arms in high frequency are thin, with less highorder side branches. Secondly, the average length of all of the side branches in primary dendrite in the two cases is calculated and shown in Fig. 9, indicating that the secondary arms in low

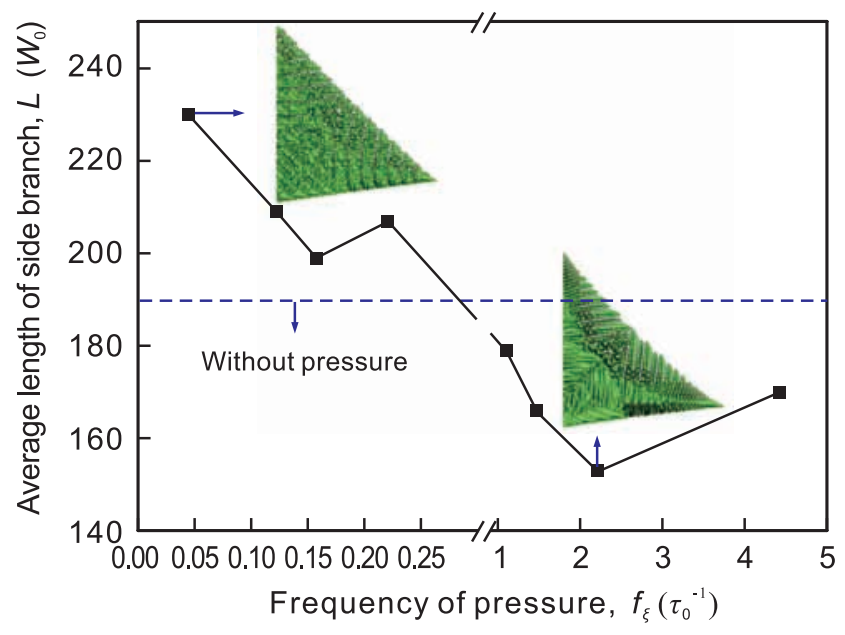

Fig. 9: Average length of side branch with different frequencies pressure 
frequency are longer than that without perturbation and much longer than that in high frequency. Thirdly, the dendrite tip without visible side branches in high frequency is much longer than that in low frequency.

Based on the formulation to calculate sidebranching frequency, i.e., Eqs. (8) and (9), the SDAS is associated with sidebranching frequency, no matter how high the frequency of pressure is, as seen from Figs. 4 and 7. Therefore, there are no great distinctions in the variation trend of SDAS with the periodic pressure in low and high frequency cases.

All the differences in sidebranching behavior can be attributed to the different modulation mechanisms in the two cases. Tip growth velocity changes synchronously with that of external pressure due to the classical Clausius-Clapeyron equation ${ }^{[25]}$. More exactly, the frequency of tip velocity is the same as that of periodic pressure in the two cases.

In the low frequency case, the oscillation of tip velocity in one period results in the formation of one side branch, indicating that sidebranching frequency is consistent with the frequency of tip velocity and the periodic pressure ${ }^{[25]}$ Therefore, in this case, the periodic pressure determines tip velocity and then modulates sidebranching directly. Especially, when the frequency of pressure is in the range of unperturbed sidebranching frequency, resonant sidebranching with unperturbed sidebranching happens, resulting in the highest sidebranching frequency (when $f_{\xi}=0.157 / \tau_{0}$ ) in this case.

In the high frequency case, there is no direct modulation of tip velocity on dendritic sidebranching since the frequency of tip velocity is too high ${ }^{[25]}$. It can be induced that there may be a bridge between tip velocity and sidebranching with much lower frequency than that of tip velocity in this case. It is supposed that there exist tiny protuberances in the dendrite tip which grow resonantly with that of tip velocity. More exactly, the frequency of tiny protuberances is consistent with that of tip velocity. Tiny protuberances are even too tiny to be visible and part of them will evolve into visible secondary arms in primary dendrite at later times. The higher the frequency of the velocity, the more tiny protuberances will be generated, and more of them evolve into secondary arms. So sidebranching frequency increases with the frequency of tip velocity until it's too high for tiny protuberances to follow. Accordingly, it can be inferred that the modulation mechanism in high frequency is that the periodic pressure modulates tip velocity which then determines the frequency of invisible tiny protuberances, and further modulates visible secondary arms indirectly.

Since sidebranching is directly modulated by periodic pressure in low frequency, and resonant sidebranching may happen, secondary arms in this case are more luxuriant, with longer average length of secondary arms than those in high frequency. This is the reason for the first and second differences in dendrite morphology. However, in high frequency, periodic pressure cannot determine sidebranching directly, but via modulating inviable tiny protuberances. So, in high frequency, the dendrite tip, which has no visible side branches but may have invisible tiny protuberances, is much longer than that in low frequency. Since tiny protuberances take part in the whole driving force, secondary arms in high frequency are less developed than those in low frequency, which also explains the first and second different phenomena.

\section{Conclusions}

Based on the detailed simulation results and analysis, the influence of periodic pressure with low frequency and high frequency on dendritic morphology and secondary arms was revealed. The results are as follows:

(1) Both in low frequency and high frequency cases, the variation trend of SDAS is inverse to that of sidebranching frequency. The lowest SDAS is obtained when perturbed by the periodic pressure with the frequency of $0.157 / \tau_{0}$ and $2.200 / \tau_{0}$ in low and high frequency cases, respectively.

(2) The average length of secondary arms changes consistently with that of sidebranching frequency when the frequency of the pressure increases. Higher sidebranching frequency indicates that more secondary arms share the whole driving force of dendrite growth, resulting in smaller driving force for each one and thus leading to less developed secondary arms.

(3) Comparisons of dendritic morphology and secondary arms are made between the low frequency case and high frequency case. Firstly, in the low frequency case, secondary arms are luxuriant especially when pressure frequency is low, with many high-order side branches stretching out. Secondly, the average length of secondary arms in primary dendrite is longer in the low frequency case than that without pressure, and much longer than that in the high frequency case. Thirdly, the dendrite tip without side branches in high frequency is much longer than that in low frequency.

(4) All the differences in dendritic morphology and sidebranching can be attributed to the different modulation mechanisms in the two cases. In the two cases, periodic pressure determines tip growth velocity of the dendrite, making it synchronized with pressure. In the low frequency case, tip velocity modulates sidebranching directly. While in the high frequency case, the frequency of tip velocity is too high to modulate sidebranching directly. The high frequency velocity influences side branches via modulating tiny protuberances in the dendrite tip, part of which evolves into side branch. In this case, the tiny protuberances take part in the whole driving force, leading to less developed secondary arms.

\section{Acknowledgement}

This work was supported by the National High Technology Research and Development Program of China (Grant No. 2018YFE0204300) and Institute Guo Qiang, Tsinghua University (Grant No. 2019GQG1010).

\section{References}

[1] Sawada T, Takemura K, Kitamura K, et al. Crystal growth by pressure control using a diamond anvil cell. Journal of Crystal Growth, 1988, 88(4): 535-536. 
[2] Sawada T, Takemura K, Shigematsu K, et al. Dynamic pressure control for solution growth and its microgravity application. Journal of Crystal Growth, 1996, 158(3): 328-335.

[3] Sawada T, Takemura K, Shigematsu K, et al. Effects of gravity On a free dendrite of $\mathrm{NH}_{4} \mathrm{Cl}$ grown by dynamic pressure control. Journal of Crystal Growth, 1998, 191(1-2): 225-233.

[4] Lacombe J, Koss M, Tennenhouse La, et al. The clapeyron effect in succinonitrile: Applications to crystal growth. Journal of Crystal Growth, 1998, 194(1): 143-148.

[5] Kar P, Lacombe J, Koss M. Velocity and radius transients during pressure nediated dendritic growth of succinonitrile. Materials Science and Technology, 2004, 20(10): 1273-1280.

[6] Koss M B, Lacombe J C, Chait A, et al. Pressure-mediated effects on thermal dendrites. Journal of Crystal Growth, 2005, 279(1-2): 170-185.

[7] Yokoyama C, Tamura Y, Nishiyama Y. Crystal growth rates of tricaprin and trilaurin under high pressures. Journal of Crystal Growth, 1998, 191(4): 827-833.

[8] Sachdeva D, Tiwari S, Sundarraj S, et al. Microstructure and corrosion characterization of squeeze cast AM50 magnesium alloys. Metallurgical and Materials Transactions B, 2010, 41(6): 1375-1383.

[9] Masoumi M, Hu H. Influence of applied pressure on microstructure and tensile properties of squeeze cast magnesium Mg-Al-Ca alloy. Materials Science and Engineering: A, 2011, 528(10-11): 3589-3593.

[10] Han Z Q, Pan H W, Li Y D, et al. Study on pressurized solidification behavior and microstructure characteristics of squeeze casting magnesium alloy AZ91D. Metallurgical and Materials Transactions B, 2015, 46(1): 328-336.

[11] Cummins H, Qian X. Dendritic sidebranching initiation by a localized heat pulse. Physical Review Letters, 1990, 64(25): 3038-3041.

[12] Muschol M, Qian X, Losert W, et al. Dendritic sidebranching with periodic localized perturbations: Directional solidification of pivalic acid-coumarin 152 mixtures. Physical Review E, 1993, 48(1): 489-499.

[13] Bouissou P, Chiffaudel A, Perrin B, et al. Dendritic sidebranching forced by an external flow. Europhysics Letters, 1990, 13(1): 89-94.

[14] Börzsönyi T, Tóth-Katona T, Buka Á, et al. Dendrites regularized by spatially homogeneous time-periodic forcing. Physical Review Letters, 1999, 83(14): 2853-2856.

[15] Börzsönyi T, Tóth-Katona T, Buka Á, et al. Regular dendritic patterns induced by nonlocal time-periodic forcing. Physical Review E, 2000, 62(6): 7817-7827.

[16] Kim S, Kim W, Suzuki T. Phase-field model for binary alloys. Physical Review E, 1999, 60(6): 7186-7197.
[17] Kim S. A Phase-field model with antitrapping current for multicomponent alloys with arbitrary thermodynamic properties. Acta Materialia, 2007, 55(13): 4391-4399.

[18] Karma A. Phase-field formulation for quantitative modeling of alloy solidification. Physical Review Letters, 2001, 87(11): 115701.

[19] Echebarria B, Folch R, Karma A, et al. Quantitative phase-field model of alloy solidification. Physical Review E, 2004, 70(6): 61604.

[20] Provatas N, Elder K. Phase-field methods in materials science and engineering. John Wiley \& Sons, 2011.

[21] Böttger B, Steinbach I, Eiken J. Multiphase-field approach for multicomponent alloys with extrapolation scheme for numerical application. Physical Review E, 2006, 73(6): 66122.

[22] Han Z Q, Han G M, Luo Alana, et al. Large-scale threedimensional phase-field simulation of multi-variant $\beta-\mathrm{Mg}_{17} \mathrm{Al}_{12}$ in Mg-Al-based alloys. Computational Materials Science, 2015, 101: 248-254.

[23] Han G M, Han Z Q, Luo A A, et al. Three-dimensional phasefield simulation and experimental validation of $\beta-\mathrm{Mg}_{17} \mathrm{Al}_{12}$ phase precipitation in Mg-Al-based alloys. Metallurgical and Materials Transactions A, 2015, 46(2): 948-962.

[24] Pan H W, Han Z Q, Liu B C. Study on dendritic growth in pressurized solidification of $\mathrm{Mg}-\mathrm{Al}$ alloy using phase field simulation. Journal of Materials Science \& Technology, 2016, 32(1): 68-75.

[25] Shang S, Guo Z P, Han Z Q. On the kinetics of dendritic sidebranching: A three dimensional phase field study. Journal of Applied Physics, 2016, 119(16): 164305.

[26] Shang S, Han Z Q, Sun W H, et al. A phase field model coupled with pressure-effect-embedded thermodynamic modeling for describing microstructure and microsegregation in pressurized solidification of a ternary magnesium alloy. Computational Materials Science, 2017, 136: 264-270.

[27] Guo Z P, Xiong S M. On solving the 3-D phase field equations by employing a parallel-adaptive mesh refinement (Para-AMR) algorithm. Computer Physics Communications, 2015, (190): 89-97.

[28] Koss M, Frei J, Giummarra C, et al. Evidence for tip velocity oscillations in dendritic solidification. Physical Review E, 2002, 65(3): 31604.

[29] Liu S, Lu S Z, Hellawell A. Dendritic array growth in the systems $\mathrm{NH}_{4} \mathrm{Cl}-\mathrm{H}_{2} \mathrm{O}$ and $\left[\mathrm{CH}_{2} \mathrm{CN}\right]_{2}-\mathrm{H}_{2} \mathrm{O}$ : Dendrite tip behavior and the origin of side arm evolution. Journal of Crystal Growth, 2002, 234(4): 751-758 